Mitteilungen der Österreichischen Geographischen Gesellschaft, 162. Jg., S. 343-368

(Annals of the Austrian Geographical Society, Vol. 162, pp. 343-368)

Wien (Vienna) 2020, https://doi.org/10.1553/moegg162s343

\title{
Migration
}

MIGRATION

\section{WANDERUNGSMOTIVE AUSGEWÄHLTER BEVÖLKERUNGSGRUPPEN IN KÄRNTEN}

\author{
Rudolf WASTL, Klagenfurt am Wörthersee* \\ Erste Einreichung / initial submission: 07/2020; revidierte Fassung / revised submission: 12/2020; \\ endgültige Annahme / final acceptance: 12/2020 \\ mit 4 Abb. und 3 Tab. im Text
}

\section{INHALT}

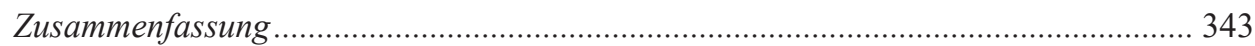

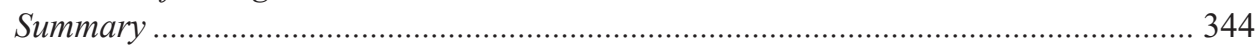

1 Forschungsthema, Charakteristika des Untersuchungsraums und relevante

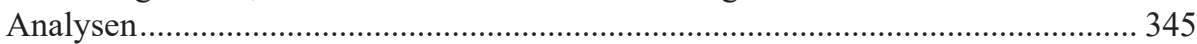

2 Fragestellung und Ziel der Studie ..................................................................... 348

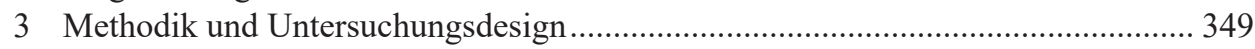

4 Motive für die Abwanderung in höhere Zentren.................................................... 353

5 Motive für die Zuwanderung aus höheren Zentren und den Verbleib im ländlichen

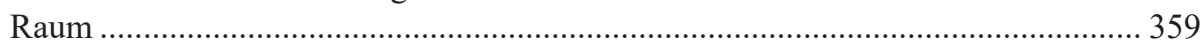

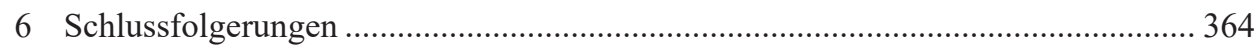

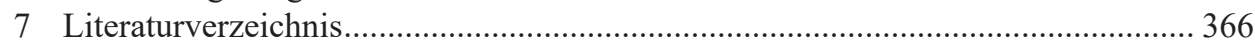

\section{Zusammenfassung}

In Regionen mit Bevölkerungsrückgang werden vor allem seitens der Politik immer wieder Maßnahmen zum Bevölkerungserhalt diskutiert. Im Hinblick darauf ist es das Ziel dieses Artikels, Verbleibe- und Wanderungsmotive besser zu verstehen, um beurteilen zu können, ob und wie weit es möglich wäre, das Wanderungsverhalten in Ab-und Zuwanderungsorten zu beeinflussen. Kärnten bietet sich als Untersuchungsraum an, weil hier

\footnotetext{
* Dr. Rudolf WASTL, Univ.-Lektor, Universität Klagenfurt, Institut für Geographie und Regionalforschung, Universitätsstraße 65-67, A-9020 Klagenfurt am Wörthersee, Austria. - E-Mail: rudolf.wastl@aau.at.
} 
schon große Teile des Bundeslandes vom Bevölkerungsrückgang betroffen sind und auch langfristig eine negative Bevölkerungsentwicklung prognostiziert ist.

In einer Untersuchungsreihe wurde daher der Frage nachgegangen, was speziell junge Berufseinsteiger, aber auch ältere Berufstätige im Allgemeinen sowie im Ruhestand befindliche Personen bewegt, ihren Wohnort zu verlegen.

Die empirische Untersuchung erfolgte durch offene Interviews mit Personen der genannten Gruppen, die retrospektiv ihre Wanderungsmotive nannten und diese entsprechend ihrer persönlichen Bedeutung gewichteten. Es standen somit qualitative und quantitative Daten von 584 Personen zur Verfügung, was eine detaillierte Auswertung erlaubte.

Das Ergebnis zeigt, dass bei Wanderungen von Berufseinsteigern in höhere Zentren berufsbezogene Motive im Vordergrund stehen und dass diese Motive in linearer Abfolge immer gewichtiger werden, je höherrangiger das Zuwanderungszentrum ist. Im Gegenzug verlieren die Motive bezüglich sozialer Beziehungen als zweitstärkste Gruppe mit zunehmender Zentralitätsstufe an Bedeutung. Versorgungs- und dienstleistungsbezogene Motive bleiben als drittwichtigste Gruppe eher konstant. Insgesamt steht für Berufseinsteiger die Arbeitsmigration im Vordergrund.

Beim Verbleib von Berufseinsteigern im ländlichen Raum oder bei der Zuwanderung von Berufstätigen und Pensionierten in diesen sowie in Mittelzentren ist es umgekehrt. Die gewichtigste Motivkategorie sind soziale Beziehungen, knapp gefolgt von der Kategorie der umfeldbezogenen Motive. Berufsbezogene sowie versorgungs- und dienstleistungsbezogene Motive haben den geringsten Stellenwert. Hier steht der Typ der „Lifestyle Migration " im Vordergrund.

Schlagwörter: Migrationsmotive, Wanderungsverhalten, Arbeitsmigration, „Lifestyle Migration“, Zuwanderungszentren, Rückwanderer, Zuwanderung, Abwanderung, Zentrale Orte, ländlicher Raum, Kärnten

\section{Summary}

\section{Migration Motives of Selected Population Groups in Carinthia}

Politicians of regions that experience a declining population size constantly discuss and search for measures to stabilise and maintain local population figures. In this regard it is the aim of this article to develop a better understanding of the motivations for staying and migrating and to be able to assess whether, and to what extent, it could be possible to influence migratory behaviour in the future. Carinthia can be deemed a suitable area for such an analysis, as large areas of the Southern Austrian province are already affected by population decline and continued negative population development is predicted for the long term.

In a series of studies and interviews, three groups - young job starters, established professionals and retirees - were asked what motivates them to relocate their residence. The empirical study was conducted through open interviews with persons of the mentioned groups. They were asked to name their motives retrospectively and to weight them according to their importance. These qualitative and quantitative data from 584 participants allowed a detailed analysis. 
The results show that employment related motives are the dominant factor for job beginners to migrate to higher-ranking central places and that these employment related motives increase in importance in correlation to the increase in urban centrality. By contrast the second strongest group of motives concerning social relationships declines in importance with in-migration to higher-ranking central places. The third strongest group of supply-and service-related motives remain rather the same. Overall, the focus is on labour migration for job beginners.

The opposite is true across all other groups. Non-migrating job starters, in-migrating employees as well as retired people in rural areas and medium-sized centres gave the greatest value to motives of social relations followed closely by environment-related motives. Job related as well as supply-and service-related motives have the lowest priority. In this case the focus is on lifestyle migration.

Keywords: Migration motives, migration behaviour, labour migration, lifestyle migration, centres of immigration, return migration, in-migration, out-migration, urban hierarchy, rural areas, Carinthia

\section{Forschungsthema, Charakteristika des Untersuchungsraums und relevante Analysen}

\subsection{Forschungsthema und Untersuchungsraum}

In weiten Teilen peripherer und strukturschwacher ländlicher Räume sind die Auswirkungen der demographischen Veränderungen mit rückläufigen Geburtenzahlen, Abwanderung junger Menschen und Alterung der Bevölkerung bereits deutlich feststellbar. Mangelnde Auslastung von Infrastruktureinrichtungen wie Verkehrssystemen, Schulen, Geschäften, medizinischer Versorgung, Einrichtungen öffentlicher Dienstleistungen und Freizeiteinrichtungen führen oft zu deren Stilllegung in kleineren Orten bzw. Zusammenlegung in zentralen Orten, sodass auch immer größere Distanzen überwunden werden müssen, um sie zu erreichen. Diese Tendenz dürfte sich fortsetzen und sogar noch verstärken.

Es gibt daher zunehmend Bemühungen, diesen Trend zumindest abzuschwächen. Die Ansätze reichen von der Schaffung zusätzlicher Arbeitsplätze in der Peripherie über die Visionen von wahlwerbenden Parteien und Zielformulierungen von Landesregierungen, die Abwanderung nicht nur aus peripheren Regionen, sondern für ein gesamtes Bundesland zu reduzieren bis zu neuerdings verstärkten Bemühungen um Rückkehrer und $\mathrm{Zu}$ wanderer.

Wesentlich für die weitere Entwicklung wird somit die Mobilität der Bevölkerung sein. Aus der gesamten Diversität der Wanderungsbewegungen (KoRDEL 2017, S. 4ff) von unidirektionalen wie der Land-Stadt-Wanderung bis zu multilokalen Lebensformen - wirken jene mit der dauerhaften Verlegung des Wohnsitzes in die ländliche Region am nachhaltigsten. Daher fokussiert diese Untersuchung auf Ab- und Zuwanderungen, wobei entsprechend der soziologischen Lebenslaufforschung (WINGENs 2020) unterschiedliche Personengruppen in wichtigen Lebens- und Berufsphasen im Zentrum stehen. 
Zur Abschätzung politischer Handlungsspielräume und Beurteilung eventuell möglicher Steuerungsmaßnahmen zur Verbesserung der Wanderungsbilanz in ländlichen Räumen und niederrangigen Zentren bedarf es eines Verständnisses dafür, welche Motive die Wanderungsentscheidung potenzieller Migrantinnen und Migranten in welcher Intensität beeinflussen. In der vorliegenden Untersuchung erfolgt dies retrospektiv.

Die oben genannte Problematik der fortschreitenden Entleerung des ländlichen Raumes zeigt sich besonders in Kärnten, das nicht nur den geringsten Bevölkerungszuwachs (0,2 \%) aller österreichischen Bundesländer von 2006 bis 2016 hatte, sondern wo auch alle Randbezirke außerhalb des Kärntner Zentralraumes (die Bezirke Klagenfurt am Wörthersee, Klagenfurt-Land, Villach und Villach-Land) einen Bevölkerungsverlust von durchschnittlich -3,3 Prozent im genannten Zeitraum aufwiesen (ÖROK 2020b).

Kärnten bietet sich zusätzlich als Untersuchungsraum an, weil es insgesamt nur einen relativ kleinen Wirtschaftsraum darstellt, in dem überdies große Teile im Norden und Westen in der ,inneren Peripherie“ Österreichs liegen, wo die positiven Kräfte der Zentralräume nicht mehr zur Wirkung kommen (vgl. SEGER 2019, S. 32). Kärnten hat traditionell eine insgesamt unterdurchschnittliche Wirtschaftsleistung mit einem Bruttoregionalprodukt pro Kopf im Jahresmittel 2015 bis 2017 von nur 84 Prozent des österreichischen Durchschnitts (ÖROK 2020a).

Die Bevölkerungsentwicklung von 2018 bis 2040 wird nur für Kärnten als einzigem österreichischen Bundesland mit einem Rückgang der Bevölkerungszahl von -2,3 Prozent prognostiziert (ÖROK 2018a). Viel dramatischer ist die Prognose für den gleichen Zeitraum für die Bevölkerung im Erwerbsalter von 20 bis 64 Jahren mit -18,7 Prozent. Dabei zeigen sich sehr starke regionale Unterschiede: In den Randbezirken des Bundeslandes (Hermagor, Spittal an der Drau, Feldkirchen, St. Veit, Wolfsberg und Völkermarkt) wird demnach der Rückgang der Erwerbsfähigen im Alter von 20 bis 64 Jahren im Mittel sogar mehr als -25 Prozent betragen - in der Landeshauptstadt Klagenfurt am Wörthersee hingegen nur -5,8 Prozent (ÖROK 2018a). Solche Prognosen verdeutlichen sehr eindrücklich die zukünftige große demographische Herausforderung und initiieren Überlegungen zu Abmilderungsversuchen seitens der Politik - unter anderem auch, das Wanderungsverhalten zu beeinflussen.

Regionale Unterschiede sind auch bei der bisherigen Bevölkerungsdynamik und der Wanderungsbilanz deutlich zu sehen. Differenziert man zum Beispiel den leicht positiven Wanderungssaldo von 0,29 Prozent des Jahres 2018 nach Raumtypen in einer ZentrumPeripherie-Abfolge, beispielsweise nach

- Oberzentren wie Klagenfurt am Wörthersee und Villach

- Mittelzentren wie den Bezirkshauptstädten

- dem zentral gelegenen ländlichen Raum mit den Bezirken Klagenfurt-Land und Villach-Land und

- dem sonstigen ländlichen Raum der Randbezirke,

so sieht man den klaren Trend von deutlichen Wanderungsgewinnen der Oberzentren bis zu leichten Wanderungsverlusten im sonstigen ländlichen Raum (vgl. Tab. 1).

Dieser Erkenntnis folgend, bezieht sich die vorliegende Untersuchung der Ab- und Zuwanderungen daher nicht auf das gesamte Bundesland, sondern auf Abwanderungen aus Kärntner Gemeinden mit keiner oder niedriger Zentralität bzw. aus Mittelzentren in höhe- 
re zentrale Orte in und vor allem außerhalb Kärntens und umgekehrt auf die Zuwanderung aus höheren Zentren in Orte niedriger oder keiner Zentralität.

\begin{tabular}{|l|c|}
\hline \multicolumn{1}{|c|}{ Raumtyp } & Wanderungsgewinn /-verlust 2018 \\
\hline Oberzentren & $+0,62 \%$ \\
\hline Mittelzentren & $+0,32 \%$ \\
\hline ländlicher Raum insgesamt & $+0,09 \%$ \\
\hline - zentraler ländlicher Raum & $+0,47 \%$ \\
\hline - sonstiger, meist strukturschwacher ländlicher Raum & $-0,16 \%$ \\
\hline
\end{tabular}

Quelle: Datengrundlage: Statistik Austria 2019a; eigene Berechnung

Tab. 1: Wanderungsbilanz für unterschiedliche Teilräume in Kärnten im Jahr 2018

\subsection{Relevante Untersuchungen}

Veränderungen der Bevölkerungszahl in einer Raumeinheit beruhen - abgesehen von der Geburtenbilanz - auf der Wanderungsbilanz und diese auf Wanderungsverlusten und Wanderungsgewinnen. Vielfach wurden bisher Wanderungsverluste als Problem thematisiert und beklagt, dass vor allem gut ausgebildete junge Menschen den peripheren ländlichen Raum verlassen. Maßnahmen, die zur Zuwanderung oder Rückwanderung motivieren sollen, wurde dagegen auch in der öffentlichen Diskussion nur ein bescheidener Stellenwert eingeräumt. Ansätze dazu gibt es zum Beispiel in einer Publikation der Österreichischen Raumordnungskonferenz, die sich mit Strategien für Regionen mit Bevölkerungsrückgang auseinandersetzt und neben Analysen auch Handlungsempfehlungen gibt. Eine der dort formulierten Empfehlungen betrifft weiterführende Motivanalysen der Zuwanderung in Regionen mit Bevölkerungsrückgang (vgl. ÖROK 2018b, S. 65). In der vorliegenden Untersuchung wird daher neben den Abwanderungsmotiven auch Verbleibe- und Zuwanderungsmotiven nachgegangen.

In einer umfassenden quantitativen Studie über Personen, die ihren Hauptwohnsitz aus Kärnten in ein anderes Bundesland verlegt haben und dabei mindesten 15 Jahre alt waren, konnten Aigner-WALDER et al. (2015) herausfinden, dass die Hauptmotive für den Wegzug aus Kärnten mit knapp der Hälfte Ausbildungszwecke sowie mit knapp über 20 Prozent der Antritt einer Arbeitsstelle und mit knapp unter 20 Prozent familiäre Gründe waren (vgl. AIGNERWALDER et al. 2015, S. 11ff). Von jenen Personen, die bereits berufstätig waren, gaben nur 12 Prozent an, ihren Lebensmittelpunkt nach Kärnten zurückverlegen zu wollen, 45 Prozent wollten dies dezidiert nicht. Dies kann als Hinweis gesehen werden, dass eine größere Anzahl von Rückkehrenden nicht zu erwarten ist. Interessant ist weiters, dass Personen mit Rückkehrabsicht als wichtigste Gründe die höhere Lebensqualität in Kärnten, soziale Bindungen und die guten Freizeitmöglichkeiten nannten, während jene, die nicht beabsichtigten zurückzukehren, überwiegend arbeitsplatzspezifische Faktoren zur Rückkehrvoraussetzung machten, wie ein ausreichendes Angebot an adäquaten Stellen, entsprechende Stellenangebote für Lebenspartnerinnen bzw. Lebenspartner oder andere Einkommensperspektiven. 
In der oben genannten Untersuchung sind die Befragungsergebnisse von Personen aus dem gesamten Bundesland zusammengefasst. Bei der Studie sind im Hinblick auf die erfolgte Abwanderung Personen befragt worden, die aus der Perspektive einer Lebenslaufforschung zwei unterschiedlichen Phasen zugeordnet werden können, nämlich jener der (meist höheren) Ausbildung und jener des Berufseinstieges. Bei den Ausbildungsmigrantinnen und -migranten ist eine vorerst vorübergehende Verlegung des Wohnortes die Ursache der Mobilität und für den später folgenden Berufseinstieg stehen dann auch noch andere Optionen offen. Bei den abgewanderten Berufseinsteigerinnen und -einsteigern war damit meist auch die Loslösung vom elterlichen Haushalt und eine Verlegung des Wohnsitzes auf unbegrenzte Zeit verbunden.

In einer weiteren Untersuchung ist Tatjana FISCHER (2015) den Hintergründen für das Bleiben, Gehen und Zurückkommen von Personen im Alter von 20 bis 29 Jahren in strukturschwachen Gemeinden der Nationalparkregion Kalkalpen in Niederösterreich im Rahmen einer schriftlichen Vollerhebung nachgegangen. Der Fokus lag hier bei den „am Land“ Lebenden, da „Ortstreue“ und „Zugezogene“ im kleinräumigen Maßstab überwogen. Sie konnte feststellen, dass die Wahl der Wohngemeinde primär von „harten“ Standortfaktoren wie Arbeitsmöglichkeiten, Basisversorgung, Freizeitangebot und Qualität des öffentlichen Nahverkehrs bestimmt wird. Erst wenn diese Grundvoraussetzungen erfüllt sind, kommen die „weichen“ Standortfaktoren zum Tragen, wie etwa das Vereinsleben, die Qualität der naturräumlichen Umgebung und das „Landleben“ per se (vgl. FISCHER 2015, S. 4ff).

Die eben genannten Untersuchungen beschäftigen sich intensiv mit dem Wanderungsverhalten junger Personen - zum einen für ein ganzes Bundesland und dessen Grenzen überschreitend und zum anderen im kleinräumigeren Maßstab und überwiegend innerhalb des ländlichen Raumes.

BARCUS und HALFACREE (2018) skizzieren Migration nicht nur als Wechsel des Wohnortes, sondern kontextualisieren sie im Zusammenhang mit Lebensläufen und Übergängen zwischen einzelnen Lebensabschnitten. Als bedeutende Übergänge, die mit Wanderungsbewegungen in Zusammenhang stehen, werden u. a. folgende beschrieben: Wanderung zur höheren Ausbildung, Verlassen des elterlichen Haushaltes, Berufseinstieg und berufliche Veränderungen, Beziehungsbildung, Wohnraumanpassung an Familiengröße, Anpassung an den Lebensstil und Pensionierung (vgl. Barcus und Halfacree 2018, S. 120).

Ihrem „Life Course“-Ansatz folgend wird der Fokus in der vorliegenden Studie auf junge Erwachsene gelegt, die das Berufsleben gerade begonnen haben, auf berufstätige Erwachsene, die aus unterschiedlichen Motiven gewandert sind, sowie Pensionierte. Da bei Ausbildungsmigrantinnen und -migranten noch offen ist, wo sie nach der Ausbildung ihren längerfristigen Wohnsitz wählen, wurden sie in die gegenständliche Untersuchung nicht aufgenommen.

\section{Fragestellung und Ziel der Studie}

Ein wesentlicher Aspekt der Studie ist es, die Wanderungsmotive jener Personengruppen nachzuvollziehen, bei denen es die größte Migrationshäufigkeit gibt und bei deren Ab- bzw. Zuwanderung die nachhaltigste Auswirkung auf Räume mit stagnierender oder negativer 
Bevölkerungsentwicklung zu erwarten ist. Die höchste Wanderungsintensität wird allgemein und auch in Österreich bei den 20- bis 30-Jährigen festgestellt und dabei sind berufsbedingte Migrationsvorgänge dominant (vgl. u. a. AIGNER-WALDER et al. 2015, S. 9; BARCUS und Halfacree 2018, S. 152f; Geist und McManus 2008, S. 288f;). Im Zusammenhang mit diesen wird in der Regel jedoch nicht nach Zielorten in unterschiedlichen Raum- oder Stadttypen im Sinn einer Hierarchie zentraler Orte differenziert. Daher wurden in der vorliegenden Studie einerseits die im Zuge des Berufseinstiegs Migrierenden differenziert nach unterschiedlichen Zielorttypen befragt. Darüber hinaus sind für den ländlichen Raum die Zuwanderer in Hinblick auf die Wanderungsbilanz besonders relevant und daher wurde andererseits diese Gruppe unabhängig von ihrer Situation im Lebenslauf in die Befragung aufgenommen. De facto waren dies einerseits Berufstätige und damit im Vergleich zu den Berufseinsteigenden deutlich Ältere und andererseits Pensionierte. Als Vergleichsgruppe wurden auch die im ländlichen Raum verbliebenen Berufseinsteiger untersucht.

Aus Untersuchungen zu Migrationsmotiven geht hervor, dass sich diese zumeist auf einen konkreten Fort- oder Zuzugsraum (vgl. Stockdale 2016; Aigner-WALdER et al. 2015; FISCHER 2015) bzw. auf die Migrationsentfernung in Kilometern (vgl. ThOMAS 2019) oder auf kein näher definiertes Migrationsziel beziehen. Eine Untergliederung der Migrationsziele nach zentralörtlichen Stufen ist darüber hinaus jedoch bedeutsam, weil die Vorstellungen und Erwartungen potenzieller Migrantinnen und Migranten an unterschiedliche Stadtgrößen und -ausstattungen geknüpft sind. Ebenso werden bei der - gegenläufigen - Stadt-Land-Wanderung Erwartungen an die Qualitäten des „Ländlichen“ und dessen Ausstattung gestellt. Daher wurden in dieser Untersuchung die Ziel- und Ausgangsorte nach zentralörtlichen Stufen strukturiert und damit auch indirekt Distanzen berücksichtigt.

Ausgehend von der Feststellung, dass sich Bevölkerungsentwicklung und Wanderungsbilanz je nach räumlicher Lage deutlich unterscheiden und theoretisch fundiert in der Lebenslaufforschung wird in der entsprechenden Literatur vor allem das Abwandern junger, gut ausgebildeter Personen als wesentlicher Faktor für Bevölkerungsverluste diskutiert. Daher widmet sich die vorliegende Untersuchung der generellen Frage, was speziell junge Berufseinsteiger, aber auch ältere Berufstätige und im Ruhestand Befindliche bewegt, den Wohnort zu verlegen, und zwar

- aus dem ländlichen Raum und niedrigeren Zentren in höhere Zentren

- aus Zentren höherer Stufe in Mittelzentren und den peripheren ländlichen Raum bzw. was sie bewegt, bewusst im ländlichen Raum zu verbleiben und wie sie dies argumentieren und gewichten.

Das Ziel ist es, Verbleibe- und Wanderungsmotive besser zu verstehen und beurteilen zu können, ob und inwieweit es möglich wäre, das Wanderungsverhalten in den Kärntner $\mathrm{Ab}-$ und Zuwanderungsorten zu beeinflussen.

\section{Methodik und Untersuchungsdesign}

Da sich die Fragestellung auf subjektive Motive und ihren Stellenwert für die Migrantinnen und Migranten selbst bezieht, wurde eine offene Befragung einschließlich einer Gewichtung durch die Probandinnen und Probanden selbst als Methode gewählt. Damit 
sollten einerseits die Motive in ihrer Formulierung durch die Befragten selbst als qualitative Daten erfasst werden, andererseits wurden durch die jeweilige Gewichtung in Prozent auch quantitative Daten generiert.

Die Befragung erfolgte in themenzentrierten Interviews und Fokusgruppen zu immer der gleichen Fragestellung über mehrere Jahre - begonnen für die Abwanderungs- und Verbleibemotive mit knapp 440 Personen in den Jahren 2013 bis 2015 und 2018 sowie für die Zuwanderungsmotive aus höheren Zentren in den ländlichen Raum mit 144 Personen in den Jahren 2016, 2017 und 2019. Durchgeführt wurden die Interviews von mehr als 170 Studierenden. ${ }^{1)}$ Die Erhebungsrunden haben sich inhaltlich nicht unterschieden und die Daten konnten damit problemlos gemeinsam ausgewertet werden.

Für diese Studie wurde Wanderung als Verlegung des Wohnortes auf unbegrenzte Zeit an einen neuen Wohnort definiert. Befragt wurden Personen, die ihren Wohnort kurz zuvor verlegt hatten. Diese Personen hatten den komplexen Abwägungsprozess für die Migration bereits abgeschlossen und konnten retrospektiv darüber Auskunft geben. Mit einer erzählgenerierenden Frage nach den Gründen für die seinerzeitige Wanderungsentscheidung wurde das Interview begonnen, um die wanderungsentscheidenden Motive zu erfahren. In einem zweiten Fragenkomplex sollten je nach Bedeutung insgesamt 100 Punkte für die zuvor genannten Motive vergeben werden, um den Stellenwert der einzelnen Motive zu gewichten. Bei der Auswertung wurden alle genannten Motive textanalytisch kodiert und zu Gruppen zusammengefasst sowie die Gewichte der Aussagen Motivgruppen zugeordnet.

Die Auswahl der Befragten erfolgte durch die Studierenden fragestellungsorientiert nach Vorgaben gemäß Tabelle 2 in ihrem weiteren Bekanntenkreis. Eine Unterstützung durch Meldeämter der Gemeinden wurde aus Datenschutzgründen von diesen abgelehnt. Die Anzahl weiblicher und männlicher Personen war annähernd gleich groß. Der Anteil der Bildungsniveaus (ohne/mit Matura und Akademiker) entsprach weitgehend der gesamtösterreichischen Verteilung.

\begin{tabular}{|l|l|l|}
\hline \multicolumn{1}{|c|}{ Abwanderungsort } & \multicolumn{1}{c|}{ Zuwanderungsort } & Lebensabschnitt \\
\hline Ländlicher Raum & Mittelzentrum & Berufseinstieg \\
\hline Ländlicher Raum und Mittelzentrum & Oberzentrum & Berufseinstieg \\
\hline $\begin{array}{l}\text { Ländlicher Raum und Mittelzentrum } \\
\text { und Oberzentrum }\end{array}$ & Großstadt & Berufseinstieg \\
\hline Großstadt & Mittelzentrum & Berufstätigkeit \\
\hline Großstadt und Oberzentrum & strukturschwacher ländlicher Raum & Berufstätigkeit \\
\hline Großstadt und Oberzentrum & strukturschwacher ländlicher Raum & Ruhestand \\
\hline
\end{tabular}

Tab. 2: Kategorisierung der befragten Personen nach $\mathrm{Zu}$ - und Abwanderungsort sowie Lebensabschnitt

\footnotetext{
1) Die Interviewdurchführung erfolgte durch Studierende der Lehrveranstaltung „Vertiefung der Humangeographie“ am Institut für Geographie und Regionalforschung der Alpen-Adria Universität Klagenfurt als praktische Übung zur empirischen Sozialforschung. Ihnen sei herzlich gedankt.
} 
Hinsichtlich der beruflichen Aspekte des „life course“ wurden drei Gruppen unterschieden: Berufseinsteiger, ältere Berufstätige und Personen im Ruhestand (vgl. Tab 2). Als Berufseinsteiger wurden junge Erwachsene definiert, die in den letzten drei Jahren vor der Befragung nach ihrem Ausbildungsabschluss (z. B. Lehre, Schule, Studium) ihren ersten Job angetreten hatten. Wanderungsverluste durch diese Gruppe haben, sowohl zahlenmäßig als auch für die Geburtenbilanz mittelfristig eine besondere demographische Auswirkung. Sie bildeten daher einen Schwerpunkt der Untersuchung. Zum Vergleich wurden Berufseinsteiger, die bewusst am Wohnort im ländlichen Raum verblieben sind, ebenfalls in Hinblick auf ihre Motive befragt.

Berufstätige und Personen im Ruhestand bilden den Großteil der aus höheren Zentren in den ländlichen Raum oder in Mittelzentren Zugewanderten. Sie sind daher hinsichtlich ihrer Wanderungsmotive besonders interessant. Bei der Befragung hat sich herausgestellt, dass bei den berufstätigen Zugewanderten der Anteil an Zurückgewanderten - also Personen, die zuvor aus diesem Raum fortgezogen waren - mit knapp 40 Prozent überraschend hoch liegt. Statistische Auswertungen über den Anteil von Zurückgewanderten an allen Zugewanderten in den Befragungsorten gibt es leider nicht. Der Wert von knapp 40 Prozent kann jedoch als Hinweis gewertet werden, dass ehemals Abgewanderte für die Zuwanderung in ländliche Räume und ihre zentralen Orte ein großes Potenzial darstellen.

Die Unterteilung der Ab- und Zuwanderungsgemeinden folgt einem zentralörtlichen Schema (vgl. Tab. 2 und Abb. 1). Damit sollen die unterschiedliche Angebotsbreite und -qualität von zentralörtlichen Einrichtungen wie Versorgungs-, Bildungs- und Freizeitangebote, aber auch Arbeitsmöglichkeiten abgebildet werden. Dieses Schema orientiert sich primär an dem Entwurf für das Sachgebietsprogramm Zentrale Orte des Amtes der Kärntner Landesregierung (Amt der Kärntner Landesregierung 2013, S. 98). Dort unterscheidet man die Oberzentren Klagenfurt am Wörthersee und Villach, die sechs Bezirkshauptstädte als Mittelzentren sowie 23 Unterzentren. Zum überwiegenden Teil waren es Mittel- und Oberzentren in Kärnten, in welche die befragten Personen gewandert sind - ein kleinerer Teil auch in die benachbarten Bundesländer.

In Kärnten endet die zentralörtliche Hierarchie mit dem Oberzentrum Klagenfurt am Wörthersee mit knapp über 100.000 Einwohnern. Um für Wanderungen in und aus größeren Landeshauptstädten, wie in vielen Fällen Graz, und vor allem in und aus Millionenstädten wie Wien oder München mit noch weit größerer zentralörtlicher Ausstattung eine Differenzierung zu ermöglichen, wird in der Analyse auch die Kategorie „Großstädte“ verwendet. Bei Wanderungen in Großstädte haben die Befragten jedenfalls das Bundesland Kärnten verlassen.

Die Mittelzentren hatten in der Dekade von 2006 bis 2016 mit durchschnittlich -2,7 Prozent eine leicht negative Bevölkerungsentwicklung (eigene Berechnung aus ÖROK 2020b). Sie spielen aber sowohl als Abwanderungsorte in höhere Zentren als auch als Zuwanderungsorte aus diesen bzw. aus dem ländlichen Raum eine wichtige Rolle.

Unterzentren werden in dieser Untersuchung dem ländlichen Raum zugeordnet. Sie haben primär eine kleinregionale Versorgungsfunktion auf unterster Ebene, jedoch nicht notwendigerweise eine Arbeitsmarktfunktion.

Migrationsvorgänge innerhalb des ländlichen Raumes - und de facto meist in die Nähe des bisherigen Wohnortes - werden der Kategorie „Verbleib im ländlichen Raum“ zugeordnet. 
Der strukturschwache ländliche Raum einschließlich seiner Unterzentren (vgl. Abb. 1) steht im Fokus der Untersuchung, weil gerade dieser die größten Bevölkerungsverluste zu verzeichnen hat (siehe Tab. 1) und weil in Kärnten versucht wurde, weitere Bevölkerungsverluste durch spezielle Wohnbauförderungsmaßnahmen in strukturschwachen Räumen abzumildern (vgl. Amt der Kärntner Landesregierung 2017).

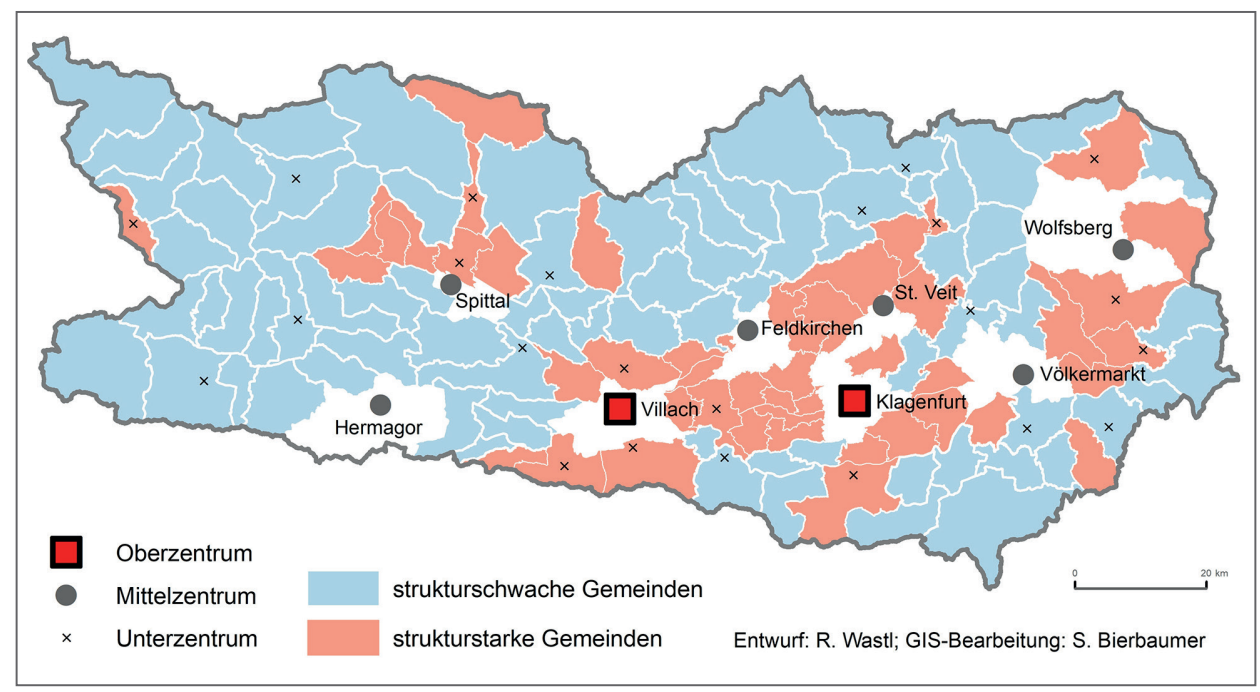

Abb. 1: Zentrale Orte und strukturschwache bzw. strukturstarke Gemeinden in Kärnten außerhalb der Ober- und Mittelzentren

Die der Untersuchung zugrunde liegende Unterscheidung strukturschwacher und strukturstarker Gemeinden außerhalb der Ober- und Mittelzentren erfolgte nach Indexpunkten auf Basis folgender Kriterien:

- Erreichbarkeit zentraler Orte unterschiedlicher Stufen gemäß ÖAMTC Routenplaner: abgestufte positive Indexpunkte für die beste Erreichbarkeit eines der folgenden Zentren im motorisierten Individualverkehr

- Oberzentrum (Klagenfurt am Wörthersee und Villach) innerhalb von 40 Minuten

- größeres Mittelzentrum (Spittal an der Drau, St. Veit an der Glan, Wolfsberg) innerhalb von 30 Minuten

- kleines Mittelzentrum (Hermagor, Feldkirchen in Kärnten, Völkermarkt) innerhalb von 20 Minuten

- Wirtschaftskraft der Gemeinde

- Steuereinnahmen der Gemeinde pro Kopf 2017 gemäß Statistik Austria 2018. Um den Mittelwert aller Gemeinden mit Ausnahme der Oberzentren (1.222 Euro) wurde eine Klasse mit null Punkten gebildet - darüber und darunter wurden positive und negative Indexpunkte vergeben.

- Arbeitsplätze je 100 Einwohner 2017 gemäß ÖROK 2019. Um den Mittelwert aller Gemeinden mit Ausnahme der Ober- und Mittelzentren (30 Arbeitsplätze je 100 
Einwohner) wurde eine Klasse mit null Punkten gebildet - darüber und darunter wurden positive und negative Indexpunkte vergeben.

- hohe Nächtigungsintensität im Tourismus gemäß Statistik Austria 2019b: Gemeinden mit 200 Nächtigungen pro Einwohner und mehr erhielten einen positiven Indexpunkt.

- Bevölkerungsentwicklung 2006 bis 2016 gemäß ÖROK 2020b: Bei einer Veränderung der Bevölkerungszahl von -2 bis +2 Prozent wurden null Punkte, bei Werten darüber bzw. darunter wurden positive bzw. negative Indexpunkte vergeben.

- Bevölkerungsentwicklung 2011 bis 2016 gem. ÖROK 2020b: bei einer Veränderung der Bevölkerungszahl von -2 bis +2 Prozent wurden null Punkte, bei Werten darüber bzw. darunter wurde ein positiver bzw. ein negativer Indexpunkt vergeben.

\section{Motive für die Abwanderung in höhere Zentren}

Bei der Auswertung hat sich gezeigt, dass die befragten Personen für ihre Migration multikausale Begründungen genannt hatten. Bei den Migrantinnen und Migranten in höhere Zentren waren es meist zwei bis vier unterschiedliche Motive, bei jenen aus höheren Zentren waren die Angaben mit drei bis sechs Motiven etwas differenzierter. Dies ist vor allem darauf zurückzuführen, dass bei letzterer Migrationsrichtung zusätzlich umfeldbezogene Motive genannt wurden.

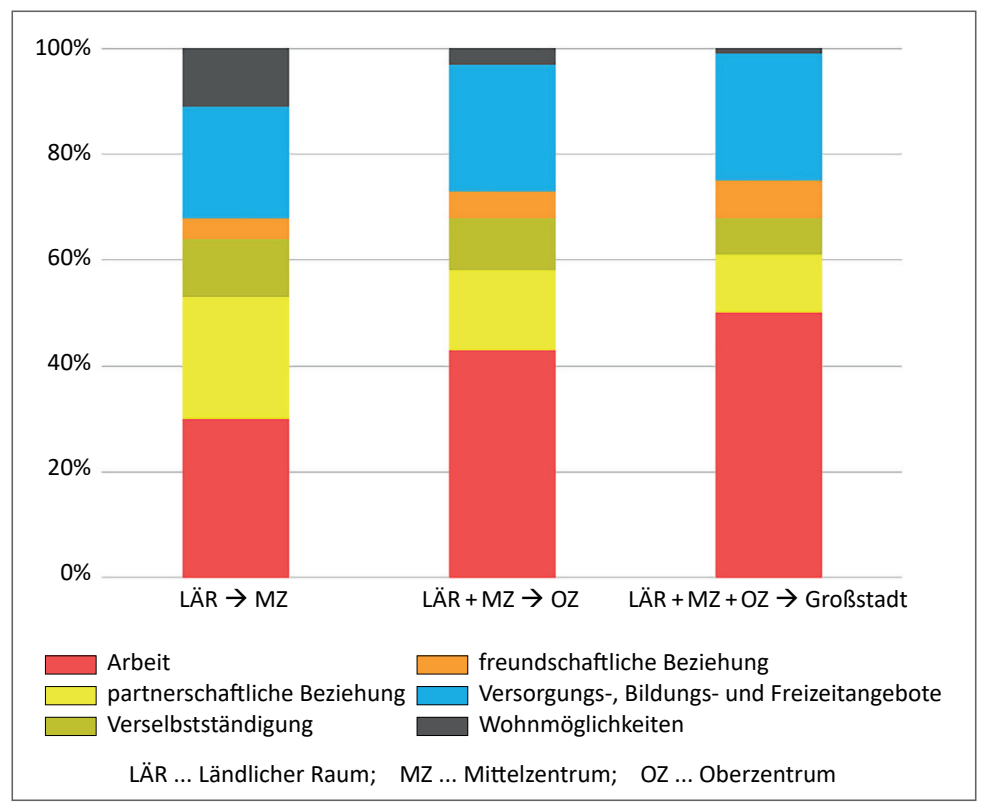

Quelle: Eigene Erhebung und Auswertung (siehe Kapitel 3); eigene Darstellung

Abb. 2: Wanderungsmotive von Berufseinsteigerinnen und Berufseinsteigern in höhere Zentren nach ihrer gewichteten Bedeutung $(n=400)$ 
Die einzelnen genannten Motive wurden zu sinngemäßen Clustern zusammengefasst, die in weiterer Folge als Motivgruppen diskutiert werden. Damit die Bandbreite der Einzelnennungen nachvollzogen werden kann, wurden jeweils typische Formulierungen für die Motive hinzugefügt.

Fasst man diese Motivgruppen zu drei Motivkategorien zusammen, werden zwei Trends sichtbar: zum einen überwiegt die Kategorie berufsbezogener Motive, gefolgt von der Kategorie sozialer Motivgruppen wie der Loslösung vom Elternhaus bzw. Verselbstständigung, der Einbindung in eine Partnerschaft und in den Freundeskreis und der Kategorie der versorgungs- und dienstleistungsbezogenen Motive. Zum anderen gibt es einen deutlichen Zusammenhang mit der Zentralitätsstufe der Wanderungsziele: je höher die Zentralitätsstufe, desto wichtiger werden berufsbezogene Motive und desto weniger bedeutend die Kategorie sozialer Motive. Die versorgungs- und dienstleistungsbezogenen Motive bleiben nahezu konstant.

\subsection{Wanderungsmotive in höhere Zentren nach Motivgruppen}

Die Gruppe berufsbezogener Motive ist für Wanderungen in Zentrale Orte und vor allem in Zentrale Orte höherer Stufe eindeutig am wichtigsten. Sie haben einen Anteil von 30 Prozent für Wanderungen aus dem ländlichen Raum in Mittelzentren, von 43 Prozent für Wanderungen aus dem ländlichen Raum und aus Mittelzentren (regionalen Zentren) in Oberzentren wie Klagenfurt am Wörthersee und Villach und sogar von 50 Prozent bei Wanderungen aus allen Raumtypen einschließlich der Oberzentren in Großstädte wie Graz oder Wien. Details zu den Motiven zeigt eine Auswahl typischer Formulierungen aus den Befragungen:

Mit Bezug auf den Zuwanderungsort: „,Arbeitsangebot war verlockend“ (im Mittelzentrum). - „,Hier (im Oberzentrum) kann ich genug Geld verdienen. “ - „Für meine Qualifikation gibt es hier (Großstadt) viele Arbeitsplätze.“ - „Ich möchte Karriere machen - das geht hier (Großstadt) besser. "

Mit Bezug auf den Abwanderungsort: „Ich hatte einen zu langen Anfahrtsweg“ (zum Mittelzentrum). - „Am Heimatort (im ländlichen Raum) gibt es einfach keinen passenden Job für mich als FH-Absolvent. “ - ,Es gibt keine g'scheite Arbeit in der Nähe“ (im ländlichen Raum). - , Vorhandene Jobs waren (im Mittelzentrum) unterbezahlt. “ - ,Weitere Aufstiegschancen waren in meinem Ort (Oberzentrum) nicht vorhanden. “

Für eine detailliertere Aussage zu berufsbezogenen Motiven wurde - auch wegen deren großer Bedeutung - bei der Befragung 2015 eine Zusatzauswertung mit detaillierteren berufsbezogenen Kategorien für 70 Personen gemacht. Dabei zeigte sich, dass bei Zuwanderern in Mittelzentren das Finden einer Arbeit ohne besondere Qualitätsansprüche, aber in guter Erreichbarkeit im Vordergrund stand und Aufstiegschancen und besseres Einkommen nachrangig waren. Umgekehrt war es bei Zuwanderern in Großstädte: bei ihnen hatte eine 
ausbildungsadäquate Arbeit einen überragenden Stellenwert und ebenso ein beruflicher Aufstieg sowie höheres Einkommen oder zumindest eine Chance dafür. Bei ihnen zeigte sich die große Differenz zwischen dem Qualifikationsprofil junger Berufseinsteiger und -einsteigerinnen und dem regionalen Arbeitsplatzangebot am Abwanderungsort am deutlichsten.

Vergleicht man die Gewichtung der berufsbezogenen Motive nach Geschlechtern, so waren deutliche Unterschiede festzustellen. Arbeit hatte generell für Männer in unseren Interviews einen deutlich höheren Stellenwert als für Frauen. Männer gewichten die Aufstiegschancen und das Vorhandensein eines Arbeitsplatzes höher. Für Frauen hatte im Unterschied dazu insbesondere eine qualifikationsentsprechende Arbeit einen höheren Stellenwert. Unterschiede nach dem Bildungsniveau der Abgewanderten waren eher gering, lediglich die Motive betreffend qualifizierte Arbeit und Aufstiegschancen hatten bei Personen mit Tertiärausbildung einen deutlich höheren Stellenwert, während Arbeit an sich und deren gute Erreichbarkeit bei Personen ohne Matura als Wanderungsgrund genannt wurde.

Die besondere Bedeutung von ausbildungsadäquaten Arbeitsplätzen und Aufstiegschancen für Abwanderer in höhere Zentren ist aus der Literatur weitgehend bekannt. Der fast modellhafte, nahezu lineare Zusammenhang mit der Zentrumshierarchie ist aber jedenfalls bemerkenswert.

Es lässt sich auch ableiten, dass Mittelzentren eine sehr wichtige Funktion für Berufseinsteiger in den regionalen Arbeitsmarkt haben und meist zumindest eine Basisausstattung an Arbeitsmöglichkeiten in akzeptabler Entfernung bereitstellen. Diese wird sichtlich von jenen jungen Erwachsenen angenommen, für die Arbeit als Wanderungsmotiv insgesamt keinen überragenden Stellenwert hat und die keine besonderen Qualitätsansprüche stellen. Es sind dies eher Männer mit niedrigerem Bildungsabschluss.

Für junge Berufseinsteigende, die in Großstädte mit einem vielfältigen und auch hochwertigen Arbeitsplatzangebot migrieren, haben berufsbezogene Wanderungsmotive einen überragenden Stellenwert. Qualifizierte Arbeit - vor allem für Frauen - und Aufstiegschancen werden für Personen mit höherem Bildungsabschluss zu dominierenden Motiven. Solche Ansprüche können in niedrigeren Zentren kaum abgedeckt werden. In Kärnten bestehen derartige Arbeitsplatzangebote am ehesten in den Oberzentren wie zum Beispiel mit „Infineon Technologies Austria“ in Villach und dem „Lakeside Science \& Technology Park“ in Klagenfurt am Wörthersee, dessen räumliches Weiterentwicklungspotenzial leider durch Raumplanungsmaßnahmen zugunsten der Wohnfunktion eingeschränkt wurde (vgl. Klagenfurt am Wörthersee 2014, S. 63ff).

Die Motivgruppe sozialer Beziehungen ist die zweitwichtigste mit einem durchschnittlichen Anteil von etwa 31 Prozent. Sie verliert bei Migrationsvorgängen in höhere zentrale Orte linear an Bedeutung, vor allem zugunsten der Gruppe berufsbezogener Motive, und lässt sich in drei Clustern von Motiven darstellen: solche für die Verselbstständigung oder Loslösung vom Elternhaus, solche betreffend partnerschaftliche Beziehungen und solche bezüglich freundschaftlicher Beziehungen.

Motive partnerschaftlicher Beziehungen waren mit 23 Prozent sehr gewichtig, vor allem für Wanderungen über kürzere Distanz in Mittelzentren. Bei Wanderungen in höhere zentrale Orte verloren sie jedoch sehr deutlich an Stellenwert (11\%). Diese Beziehungen 
sind für Personen ohne Matura deutlich wichtiger als für andere, wobei geschlechtsspezifische Unterschiede sehr gering sind. „Ich bin zum Freund (im Mittelzentrum) gezogen.“ „Meine Partnerin wohnt schon in dieser Stadt“ (Oberzentrum).

Motive für die Verselbstständigung wurden mit abnehmendem Gewicht von 11 Prozent bei Wanderung in Mittelzentren und 7 Prozent in Großstädte genannt. Der beabsichtigte Verselbstständigungsprozess junger Erwachsener wurde beispielsweise wie folgt formuliert: „Musste am Hof mitarbeiten“ (im ländlichen Raum). „Hier (im Oberzentrum) stehe ich zu viel unter dem Einfluss meiner Eltern." Der Wunsch nach Loslösung vom Elternhaus wurde vor allem von Frauen und Personen ohne Matura aus dem ländlichen Raum relativ oft genannt, er wurde mit zunehmender Zentralität der Zuwanderungsorte von Männern und Personen mit tertiärer Ausbildung immer seltener hergestellt: „Ich möchte mein eigenes Leben führen und mit dem Freund allein sein“ (im Mittelzentrum). „Dort (im Oberzentrum) kontrolliert mich niemand mehr.“ „Wollte weg von zuhause und das Leben in der Stadt ausprobieren.“ „Wollte selbstständig sein, Sachen allein erledigen und unabhängig sein.“"

Motive freundschaftlicher Beziehungen hatten mit 4 Prozent bis 7 Prozent eher wenig Gewicht - bei einer höheren Bedeutung bei Migration in höhere Zentren und für Männer mit tertiärer Ausbildung. „Ich habe Freunde dort (im Oberzentrum), um Anschluss zu finden.“ „Meine Freunde sind alle abgewandert - hier (im Oberzentrum) sind viele von ihnen.“

Die Gruppe der versorgungs- und dienstleistungsbezogenen Motive beinhaltet nicht nur Motive mit Bezug auf die Versorgungs-, Bildungs- und Freizeitangebote, sondern auch jene mit Bezug auf Wohnmöglichkeiten. Die gesamte Motivkategorie wurde von den Migrantinnen und Migranten in Mittelzentren mit 32 Prozent vergleichsweise hoch gewichtet - sie sank bei der Migration in Großstädte bis auf 25 Prozent.

Die Bandbreite der Motive mit Bezug auf die Versorgungs-, Bildungs- und Freizeitangebote wird durch folgende Auswahl typischer Formulierungen aus den Befragungen abgesteckt:

Mit Bezug auf die Wanderungsziele: „,Hier (im Mittelzentrum) bekomme ich alles auf einem Fleck - brauche nicht weit einkaufen zu gehen. “-, Mehr Möglichkeiten für Shopping und Party“ (im Oberzentrum). - „In Wien geht die Post ab - ich brauch 'das. “ - ,Es gibt perfekte Angebote zur Fortbildung “ (in der Großstadt).

Mit Bezug auf die Abwanderungsorte: , In unserer kleinen Stadt (Unterzentrum im ländlichen Raum) ist ja nichts los - kulturell und nicht einmal g'scheite Lokale. " - „Hier (im ländlichen Raum) gibt es keine Bildungsangebote. “ - ,Wir sind weit weg (im ländlichen Raum) - man braucht ewig, um etwas Vernünftiges einzukaufen. “-,Zum Einkaufen und Fortgehen war der Weg einfach zu weit."

Die genannten Motive von Zuwanderern aus dem ländlichen Raum in Mittelzentren sind offensichtlich von deutlichen Defiziten in der Basisversorgung und im Freizeitangebot im Abwanderungsort geprägt. Durch die Wanderung zumindest in Mittelzentren mit einem breiteren, wenn auch nicht sehr spezialisierten Ausstattungsangebot dürften diese Defizite regional ausgeglichen werden können. Mittelzentren haben offensichtlich auch im Bereich 
der infrastrukturellen Ausstattung eine besondere Bedeutung und wirken stabilisierend für den ländlichen Raum.

Bei Wanderungen in Zentren höherer Stufe werden die auf das Wanderungsziel gerichteten Motive immer wichtiger, während jene, die sich auf die Abwanderungsorte beziehen, massiv an Gewicht verlieren. Das Versorgungs-, Bildungs- und Freizeitangebot in Oberzentren und Großstädten ist für junge Berufstätige sichtlich sehr begehrenswert, während die diesbezüglichen Defizite in den Abwanderungsorten nur noch einen untergeordneten Stellenwert haben. Die Großstädte bieten eine große Vielfalt und Qualität der urbanen Einrichtungen und damit einen ereignisreichen und urbanen Lebensstil mit vielen Optionen, wie sie selbst in Mittel- und Oberzentren, die über gute Infrastrukturen verfügen, nicht in Anspruch genommen werden können.

Für Frauen ist das Versorgungs-, Bildungs- und Freizeitangebot ein gewichtigeres Wanderungsmotiv als für Männer. Sie betonen die infrastrukturellen Defizite im ländlichen Raum und in den Mittelzentren und die gute Verkehrsinfrastrukturausstattung in Großstädten besonders. Für Personen ohne Matura sind das mangelnde Freizeitangebot im ländlichen Raum und die große Distanz, um Güter des täglichen Bedarfs zu besorgen, verstärkte Motive, in Mittelzentren zu übersiedeln. Personen mit höherer Bildung nennen häufig das breitere Fortbildungsangebot von Oberzentren und Großstädten.

Motive mit Bezug auf die Wohnmöglichkeiten waren für Wanderungen aus dem ländlichen Raum in Mittelzentren mit 11 Prozent noch ein gewichtiges Motiv, wobei die Defizite im ländlichen Raum wesentlich stärker betont wurden als die Vorteile der Mittelstädte: „Hier (im ländlichen Raum) findet man ja keine brauchbare Wohnung.“ „Hier (im Mittelzentrum) gibt es mehrere Wohnmöglichkeiten." Für Wanderungsvorgänge in höhere Zentren spielt das Wohnungsangebot als Motivgruppe fast keine Rolle.

\subsection{Wanderungsmotive und ihr Bezug zu Abwanderungs- oder Zuwanderungsorten}

In einem weiteren Bearbeitungsschritt wurde ausgewertet, ob sich die genannten Motive eher auf den Abwanderungsort mit negativer Assoziation oder eher auf das Wanderungsziel mit positiven Gefühlen und Erwartungen beziehen. Das Ergebnis zeigt, dass die Wanderungsmotive mit etwa drei Viertel ihrer Gewichtung ganz klar auf die Wanderungsziele ausgerichtet sind (Tab. 3).

\begin{tabular}{|l|c|c|}
\hline \multirow{2}{*}{ Wanderungsziel } & \multicolumn{2}{|c|}{ Gewichtete Wanderungsmotive } \\
& zielortbezogene Motive & abwanderungsortbezogene Motive \\
\hline Mittelzentrum & $69 \%$ & $31 \%$ \\
\hline Oberzentrum & $78 \%$ & $22 \%$ \\
\hline Großstadt & $83 \%$ & $17 \%$ \\
\hline
\end{tabular}

Tab. 3: Gewichtung der Motive für die Wanderung in höhere Zentren durch Berufseinsteigerinnen und Berufseinsteiger $(n=400)$ 
In diesem Kontext wird auch ein klarer Zusammenhang zwischen der Motivgewichtung und der Zentralitätsstufe der Zuwanderungsorte sichtbar: je höher die Zentralitätsstufe, desto stärker überwiegt der Bezug zum Wanderungsziel und erreicht bei Großstädten über 80 Prozent. Damit wird auch deutlich, dass die wahrgenommenen und artikulierten Defizite im Bereich der Abwanderungsorte nur einen sehr kleinen Anteil an den Abwanderungsmotiven ausmachen und daher auch allfällige Maßnahmen zur Reduktion dieser Defizite und damit zur Verhinderung der Abwanderungsbereitschaft kaum bis gar nicht erfolgreich sein dürften.

\subsection{Bedingungen zum Verbleib am Abwanderungsort}

Bei einer Fokusgruppe mit 34 Migrantinnen und Migranten aus dem ländlichen Raum, die in Großstädte abgewandert sind, wurde auch die Frage nach Rahmenbedingungen gestellt, unter denen die Befragten damals nicht weggezogen wären. Für den größten Teil (37 \%) wäre eine ausbildungsadäquate, qualifizierte Arbeit in guter Erreichbarkeit ein Grund gewesen, nicht abzuwandern. Dies betraf vor allem Personen mit akademischem Berufsabschluss. Persönliche familiäre Gründe (wie eine Partnerschaft) wurden mit 25 Prozent am zweithäufigsten genannt, überwiegend von Frauen und Nichtakademikern und Nichtakademikerinnen. 21 Prozent der Befragten und dabei vorwiegend Männer gaben an, dass sie unter allen Umständen weggezogen wären. Gute Mobilität (öffentlicher Verkehr oder Führerschein und verfügbarer Pkw) wäre vor allem für Frauen auch noch eine Rahmenbedingung für den Verbleib gewesen.

Bessere Wohn- und Freizeitmöglichkeiten oder günstigere Wohnungs- oder Wohnbaufinanzierung im ländlichen Raum wurden als Bleibegründe nicht genannt. Ebenso waren Umweltqualität und emotionale Ortsbezogenheit für Personen, die den Abwägungsprozess zwischen Bleiben oder Gehen zugunsten des Weggehens entschieden hatten, kein Thema mehr. Offensichtlich kommen die letztgenannten Motive erst dann zum Tragen, wenn eine adäquate Arbeitsmöglichkeit sichergestellt ist oder keine Arbeit mehr gesucht wird. Dies trifft meist erst in einer späteren Lebensphase zu. So gesehen sind die politischen Möglichkeiten, junge Erwachsene - vor allem jene mit hoher Berufsqualifikation in ländlichen Räumen zu halten, sehr eingeschränkt. Auch spezielle Wohnbauförderungsmaßnahmen, wie sie in Kärnten eingeführt wurden, dürften daran nichts ändern.

\subsection{Resümee für die Migration von Berufseinsteigern in höhere Zentren}

In der vorliegenden Untersuchung hat sich deutlich gezeigt, dass berufsbezogene Motive am wichtigsten sind und es konnte auch der quantitative Nachweis erbracht werden, dass diese Motive in linearer Abfolge immer gewichtiger werden, je höherrangiger das Zuwanderungszentrum ist. Sie können bei Großstädten sogar 50 Prozent des gesamten Motivbündels erreichen. Im Gegenzug verlieren die Motive sozialer Beziehungen als zweitstärkste Gruppe mit zunehmender Zentralitätsstufe an Bedeutung. Versorgungs- und dienstleistungsbezogene Motive als drittwichtigste Gruppe nehmen mit zunehmender Zentralität nur leicht zu. 
Für Migrationsvorgänge aus dem ländlichen Raum in Mittelzentren sind die Erreichbarkeit einer Arbeit an sich, die gute Erreichbarkeit einer Basisausstattung an Versorgungsund Dienstleistungen sowie familiär-partnerschaftliche Beziehungen und die bessere Möglichkeit der Verselbstständigung wichtige Motive, die sich auch häufig auf Defizite der Abwanderungsorte beziehen. Mittelzentren haben zwar meist selbst eine leicht negative Bevölkerungsentwicklung, stellen aber mit ihren Arbeitsplätzen und Infrastrukturen durchaus attraktive Zuwanderungsorte aus ländlichen Räumen dar und fungieren damit als Stabilisatoren vor allem in Regionen mit Bevölkerungsrückgang (vgl. ÖROK 2018b, S. 15ff).

Bei Migrantinnen und Migranten in Oberzentren und vor allem Großstädte sind die Motive wesentlich stärker auf ausbildungsadäquate Arbeit, berufliche Aufstiegsmöglichkeiten, ein interessantes und vielfältiges Angebot an Dienstleistungen sowie Bildungs- und Freizeitmöglichkeiten ausgerichtet, jedoch weniger auf familiäre Bindungen und Verselbstständigung. Ihre Motive beziehen sich zum größten Teil auf die positiven Aspekte der Wanderungsziele. Dies zeigt auch, dass es sich nicht um eine ausschließlich berufsbedingte Wanderung handelt, sondern eine deutliche Überlappung mit Elementen einer „Lifestyle Migration“ besteht, wenn junge Berufseinsteigende, die sich oft in einer Lebensphase mit wenig familiären Verpflichtungen und großer räumlicher Flexibilität befinden, aus einer überwiegend ländlich geprägten und für sie durchaus mit einer empfundenen Enge und deutlichen Defiziten belasteten Umgebung in ein urbanes, großstädtisches Umfeld migrieren, das ihnen viele Möglichkeiten zur beruflichen und persönlichen Entwicklung verspricht (vgl. u. a. Barcus und Halfacree 2018, S. 198ff; Thomas 2019, S. 3).

Die gesammelten Motive geben wenige Ansatzpunkte, wie man die Abwanderung von Berufseinsteigenden aus ländlichen Räumen bzw. aus Kärnten insgesamt steuern oder gar verhindern könnte. Die Mittelzentren und vor allem die beiden Oberzentren Klagenfurt am Wörthersee und Villach in Kärnten haben als Zuwanderungsorte eine stabilisierende Wirkung. Ihre Ausstattung mit attraktiven Arbeits- und Bildungsmöglichkeiten sowie Einrichtungen für einen urbanen Lebensstil dürfte einen leicht dämpfenden Einfluss auf die Abwanderung haben.

\section{Motive für die Zuwanderung aus höheren Zentren und den Verbleib im ländlichen Raum}

Bei der Wanderungsrichtung aus Oberzentren und Großstädten in Mittelzentren und den peripheren ländlichen Raum hat sich im Rahmen der vorliegenden Untersuchung eine zusätzliche Kategorie mit Umweltmotiven gezeigt, sodass insgesamt vier Motivkategorien erkennbar sind. Die untersuchten Migrationsvorgänge beziehen sich hier sowohl einerseits auf die Zuwanderung von Berufstätigen und Pensionierten aus höheren Zentren in Mittelzentren und den ländlichen Raum als auch andererseits auf den Verbleib von Berufseinsteigern in diesem.

Als gewichtigste Motivkategorie ergab die Befragung die der sozialen Motive mit durchschnittlich 40 Prozent, knapp gefolgt von der Kategorie der umfeldbezogenen Motive mit durchschnittlich 35 Prozent - einer Kategorie, die bei Migration in höhere Zentren überhaupt keine Rolle spielt. Berufsbezogene Motive standen bei den Berufstätigen mit 
durchschnittlich 18 Prozent an dritter Stelle. Diese Gewichtung ist nicht einmal halb so groß wie bei Migrationen in höhere Zentren und im Zusammenhang mit der großen Bedeutung umfeldbezogener Wanderungsmotive ein klarer Hinweis auf eine „Lifestyle Migration“. An vierter Stelle kommen versorgungs- und dienstleistungsbezogene Motive mit durchschnittlich 12 Prozent, die damit ebenfalls nicht einmal halb so gewichtig sind wie bei der Wanderung in höhere Zentren.

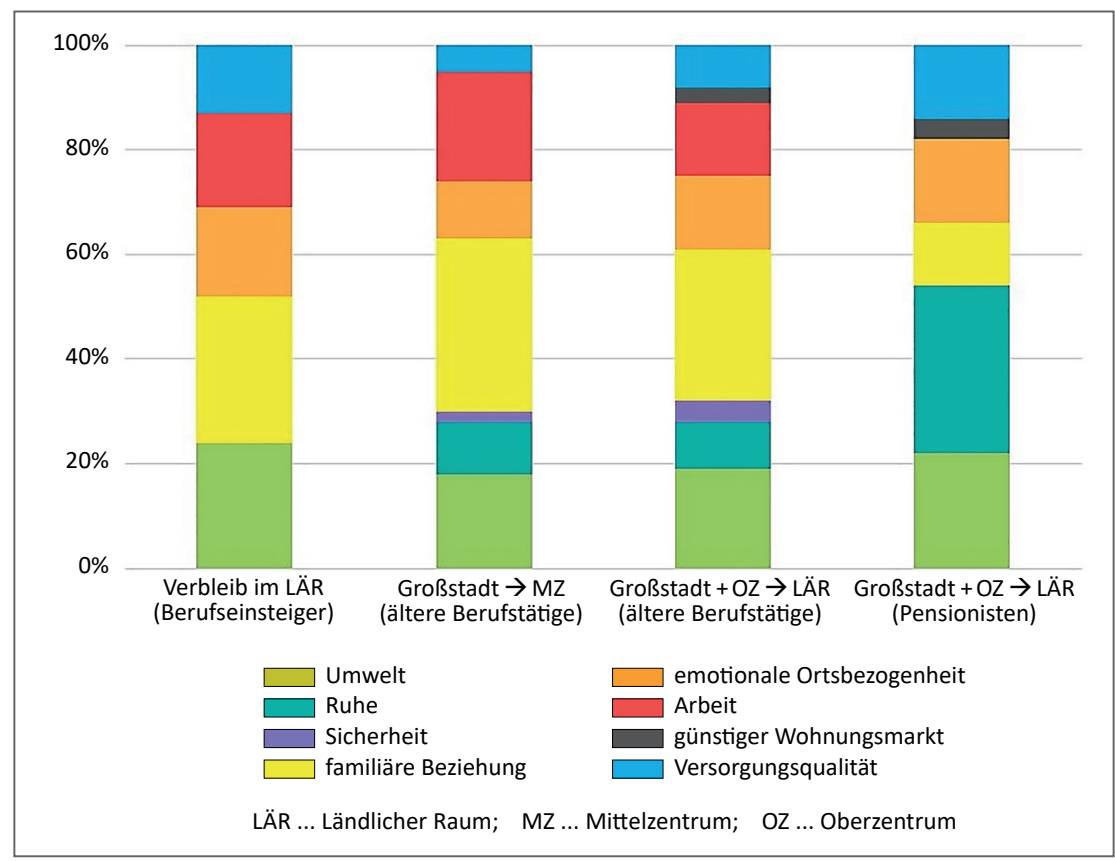

Quelle: Eigene Erhebung und Auswertung (siehe Kapitel 3); eigene Darstellung

Abb. 3: Motive für die Zuwanderung aus höheren Zentren in niedrigerrangige Zentren und den ländlichen Raum sowie für den Verbleib im ländlichen Raum nach ihrer gewichteten Bedeutung $(\mathrm{n}=184)$

\subsection{Wanderungsmotive aus höheren Zentren und Verbleibemotive im ländlichen Raum nach Motivgruppen}

In der Gruppe der umfeldbezogenen Motive lassen sich drei Cluster zusammenfassen: die umweltbezogenen, die ruhebezogenen und die sicherheitsbezogenen Motive. Sie haben bei den Berufstätigen den zweithöchsten Stellenwert von allen Motivgruppen mit 24 bis 32 Prozent und knapp über 50 Prozent bei den Pensionierten. Innerhalb der Gruppe umfeldbezogener Motive sind jene, die sich auf die Umwelt beziehen, die bedeutendsten und weitgehend unabhängig von Wanderungsbewegungen oder dem Verbleib im ländlichen Raum und auch weitgehend unabhängig von beruflicher Aktivität. Die hohe Umweltqualität wird 
offensichtlich als derart anziehend und eng mit dem ländlichen Raum sogar einschließlich seiner regionalen Zentren, den Mittelzentren, gesehen, dass sich die Formulierungen der Probandinnen und Probanden ausschließlich auf die Wanderungsziele bezogen.

Mit „Umwelt“ werden die Bereiche Natur, Landschaft, das Grüne, Luftqualität sowie die Sportmöglichkeiten im Freien angesprochen - ebenfalls die fallweisen Nennungen der guten Lage Kärntens für die Erreichbarkeit des „Südens“, wie die folgenden Formulierungen zeigen:

„,Warum soll ich weggehen, wenn es da so schön ist?“ „Meine Kinder sollten in der Natur aufwachsen! “ „Die idyllische Landschaft gefällt mir. “ „Die Luftqualität ist hier viel besser. “ „Das Leben am Land hat viel mehr Qualität. Ich muss nicht erst kilometerweise fahren, um in einem überfüllten Park zu sein. “ „Hier werden viele Sportmöglichkeiten (im Freien) geboten. “ „, Kärnten liegt so zentral, ich bin sofort im Süden, z. B. in Slowenien, Kroatien oder in Italien. “

Da die Probandinnen und Probanden sehr häufig neben der Umwelt auch die Ruhe im peripheren ländlichen Raum als Motiv beschrieben haben, wurde sie gesondert ausgewertet. Mit der Ruhe wird nicht nur ein Weniger an Verkehrslärm gemeint, sondern auch ein Teil typisch ländlicher Lebenswelt umschrieben, wie ein erholsames, weniger hektisches und beschaulicheres Leben, das sich aber nicht nur auf den peripheren ländlichen Raum, sondern auch noch auf die Mittelzentren bezieht. Nur Zugewanderte aus höheren Zentren nannten ruhebezogene Motive und bewerteten sie sehr unterschiedlich. Bei Berufstätigen lag die Gewichtung bei etwa 10 Prozent von allen Motiven - bei Pensionierten war sie mit 32 Prozent die höchste von allen. Hier kommt die Lifestylekomponente speziell in der Lebensphase der Pension deutlich zum Ausdruck.

Im ländlichen Raum verbliebene Berufseinsteiger haben dagegen die Ruhe als Motiv für ihr Verbleiben im ländlichen Raum nicht gesondert genannt - wohl aber die Umweltqualität und speziell die landschaftliche Schönheit. Die ruhebezogenen Motive wurden bei den Wanderungen aus höheren Zentren sowohl mit Bezug auf den Zuwanderungsort als auch den Abwanderungsort genannt, in dem das laute, hektische und stressige Stadtleben als abstoßendes Wanderungsmotiv angesprochen wurde. Eine Auswahl typischer Formulierungen zeigt Details zu den ruhebezogenen Motiven:

Mit Bezug auf die Wanderungsziele: „Hier ist es ruhig und erholsam. “- „Ich genieße die Ruhe und Besinnlichkeit. “- „Hier ist es stressfrei und weniger hektisch. “ - „Hier ist es ruhiger, kaum Autolärm und man kann bei offenem Fenster schlafen. “

Mit Bezug auf die Abwanderungsorte: „Der Straßenlärm war eine Zumutung. “ „Over 20 years in a big city was enough; traffic was terrible. “- „In Graz ist alles so laut und sehr schnelllebig - ich konnte nie zur Ruhe kommen. “- „Das unruhige und stressige Stadtleben hat mich genervt. "

In die Gruppe umfeldbezogener Motive sind auch jene der gefühlten Sicherheit eingeordnet. Diese bemerkenswerten Motive wurden erst bei den Befragungen 2017 und 2019 und 
nur von berufstätigen Zugewanderten (überwiegend von Männern) genannt - nicht jedoch von Verbliebenen im ländlichen Raum und auch nicht von Personen im Ruhestand. Diese sicherheitsbezogenen Motive beziehen sich auf Kriminalität, Ausländer sowie auf Gefahren des Straßenverkehrs und sind mit 3 Prozent an allen gewichteten Motiven für die beiden betroffenen Personengruppen gering. Sie werden sowohl mit Ausgangspunkt und Endpunkt der Migration in Verbindung gebracht:

Mit Bezug auf die Zuwanderungsziele: „, Weniger Kriminalität, nicht so viele ausländische Einwanderer. “ - „Hier kann man noch die Haustür offenlassen, ohne Angst haben zu müssen, dass jemand einbricht oder etwas stiehlt. “ - „, Geringere Gefährdung im Straßenverkehr für die Kinder. “

Mit Bezug auf die Abwanderungsorte: „In Wien gab es einen höheren Ausländeranteil, was mich sehr verunsicherte. “ - „, Die Kriminalität in der Großstadt ist viel höher."

Die Gruppe sozialer Motive ist die gewichtigste mit einem Anteil von über 40 Prozent an allen Motiven bei Berufstätigen, unabhängig davon, ob sie im ländlichen Raum verblieben oder aus höheren Zentren zugewandert sind. Bei Pensionierten ist der Anteil mit 28 Prozent deutlich geringer. Bei dieser Motivgruppe haben sich zwei Cluster ergeben. Einerseits die familienbezogenen Motive und andererseits eine emotionale Ortsbezogenheit.

Familiäre Beziehungen bildeten mit etwa 30 Prozent die gewichtigsten Motive von allen, sowohl für aktiv im ländlichen Raum Verbliebene als auch für Zuwanderungen Berufstätiger aus höheren Zentren. Bei Letzteren dürfte der hohe Anteil von Rückwanderern mit knapp 40 Prozent zur hohen Gewichtung beitragen. Bei Personen im Ruhestand mit sehr geringem Anteil an Zurückgewanderten liegt die Gewichtung nur bei 12 Prozent. Insgesamt spielen familiäre Beziehungen für Zuwanderungen in den ländlichen Raum eine höhere Rolle als bei Abwanderungen aus diesem.

Motivformulierungen waren unter anderem: „Ich bin zu meiner Freundin gezogen. “ „My wife was from here. " „Ich habe hier den Hof meiner Großeltern geerbt. “ „Meine Familie und Freunde leben hier in der Nähe. “ „Ich muss mich ja um meine Eltern kümmern. “

Die Motive emotionaler Ortsbezogenheit wurden von allen Befragten mit etwa 15 Prozent gewichtet und umfassten einerseits positive Erinnerungen von Zurückgewanderten und auch den Wunsch, dass ihre Kinder - so wie sie - im ländlichen Umfeld aufwachsen sollten und andererseits das typische Landleben mit seinem aktiven Vereinsleben, den engeren Kontakten zu den Leuten im Ort und dem freundlichen und hilfsbereiten Umgang miteinander in etwas entspannterer Atmosphäre. Die Gewichtung dieser Motive war bei Zuwanderungen in periphere ländliche Orte nur unwesentlich höher als bei Zuwanderungen in Mittelzentren.

Typische Beschreibungen waren: „Meine Frau und ich sind hier aufgewachsen. Unsere Kinder sollen auch hier groß werden dürfen. “ - „Die Leute hier sind 
freundlicher und herzlicher. “- „Die Menschen hier sind sehr hilfsbereit und haben keinen Stress. “ - ,Wir haben engeren Kontakt zu unseren Nachbarn. “- ,Es gibt auf dem Dorf ein starkes Vereinsleben - das bindet einen. “

Berufsbezogene Motive spielten bei allen berufstätigen Befragten mit meist knapp 20 Prozent eine bedeutende Rolle - auch wenn sie im Vergleich zu abgewanderten Berufseinsteigenden anders gelagert sind. Es stehen nicht die ausbildungsadäquaten Jobs in guter Erreichbarkeit und Aufstiegschancen im Vordergrund, sondern die Verfügbarkeit eines Arbeitsplatzes für sich selbst und/oder Familienmitglieder bzw. die Selbstständigkeit vor allem bei der Übernahme eines landwirtschaftlichen oder anderen Betriebes. Auch Wohlfühlmotive im Beruf wie geringere Hektik oder freundlichere Personen im ländlichen Umfeld spielen eine Rolle.

Typische Motivformulierungen waren: ,, Ich habe hier (im ländlichen Raum) Arbeit gefunden. “ - ,Mein Mann hat hier (im Mittelzentrum) Arbeit gefunden. “- „Ich habe den elterlichen Betrieb übernommen “ (im ländlichen Raum). - „Das Team ist viel kleiner als in Wien, der Arbeitsalltag weit weniger hektisch, die Patienten freundlicher und alle verstehen Deutsch“ (im Mittelzentrum). - ,Habe mit meinem Freund eine Bar eröffnet" (im Mittelzentrum).

Versorgungs- und dienstleistungsbezogene Motive sind mit durchschnittlich 12 Prozent gewichtet und beziehen sich überwiegend auf den günstigen Wohnungsmarkt. Dieser wurde überwiegend von Verbliebenen im ländlichen Raum und von Pensionierten genannt wie: „Hier (im Mittelzentrum) sind die Mietpreise günstig.“ „Für wenig Geld bekommt man eine traumhafte Wohnmöglichkeit (im ländlichen Raum).“ „Wir konnten uns hier (im ländlichen Raum) ein Haus leisten.“

Bei der Versorgungsqualität werden ebenfalls die günstigen Kosten hervorgehoben, aber auch das Vorhandensein einer Basisversorgung wird von Pensionistinnen und Pensionisten positiv angemerkt: „Die Lebenskosten sind hier (im ländlichen Raum) günstiger.“ „Die Schulqualität ist besser (im Mittelzentrum).“ „Es gab ein sehr gutes Angebot an Kindergartenplätzen (im ländlichen Raum).“ „Hier finde ich sogar einen exzellenten Arzt und was ich dringend brauche (im ländlichen Raum).“

\subsection{Unterschiede bei den Motivgewichtungen}

Bei Verbleib im oder Zuwanderung in den ländlichen Raum und in Mittelzentren gab es teilweise geschlechtsspezifische Unterschiede. So haben etwa Männer die Bereiche Beruf und Wohnen höher bewertet - Frauen dagegen die Bereiche Ruhe, Umwelt und vor allem beim Verbleib im ländlichen Raum die familiären Beziehungen. Hier sind traditionelle Geschlechterrollen deutlich sichtbar.

Unterschiede bei der Motivgewichtung gab es zwischen Rückgewanderten und neu Zugewanderten. Rückgewanderte haben familiäre Motive mit 15 Prozent und ruhebezogene Motive mit 5 Prozent höher gewichtet als neu Zugewanderte. Letztere bewerteten 
die Kategorien Umwelt, Ruhe und Beruf mit 5 bis 10 Prozent höher bewertet als Rückgewanderte.

Gewichtungsunterschiede zwischen Abgewanderten aus Großstädten und aus Oberzentren ergaben sich nur marginal - ebenso wie jene zwischen Zuwanderung in Unterzentren oder in Dörfer des strukturschwachen ländlichen Raumes.

Generell lässt sich auch festhalten, dass die Abwanderung aus höheren Zentren von zielortbezogenen Motiven wesentlich stärker dominiert wird als die Zuwanderung in höhere Zentren.

\subsection{Resümee für die Migration aus höheren Zentren}

Die Gewichtung der Motive zeigt zwei Schwerpunkte: Für Personen im Berufsleben mit etwa 40-prozentigem Anteil an Rückgekehrten erwiesen sich die sozialen Motive als die wichtigsten und zusammen mit den umfeldbezogenen Motiven erreichten sie 75 Prozent. Damit stellen vor allem ehemals Abgewanderte, die noch einen familiären oder emotionalen Bezug zu ihrem Abwanderungsraum haben, ein großes Potenzial für künftige Rückwanderung dar, das mehr als bisher genutzt werden könnte. Ähnliches gilt für Pensionierte mit zwar geringeren familiären Bindungen, jedoch überaus gewichtigen umfeldbezogenen Motiven. Beide Gruppen bewerten die Lebensqualität überaus hoch und diese Migrationsvorgänge sind durchaus als „Lifestyle Migration“ zu bezeichnen.

Aus der Literatur ist bekannt, dass „Lifestyle Migrantinnen und Migranten“ die besondere Lebensqualität am Zuwanderungsort meist im ländlichen Milieu höher bewerten als die Möglichkeit finanzieller und beruflicher Besserstellung (vgl. u. a. O`REILly 2014; Gruber et al. 2017, S. 17; Barcus und Halfacree 2018, S. 184). Die Ergebnisse der vorliegenden Studie bestätigen dies eindrücklich. Selbst die berufstätigen Zugewanderten haben sowohl die Gruppe der umfeldbezogenen Motive als auch jene der sozialen Motive etwa doppelt so stark gewichtet wie die berufsbezogenen Motive.

\section{Schlussfolgerungen}

Ländliche Regionen im Süden Österreichs und vor allem in Kärnten verlieren laufend an Bevölkerung und es wird immer wieder versucht, Strategien zur Abmilderung der Bevölkerungsverluste und ihrer Auswirkungen zu entwickeln. Sehr viel wird über die negative Wanderungsbilanz diskutiert und vielfach wird ein wesentlicher Grund in der Abwanderung junger Erwachsener gesehen, die entweder zur Ausbildung oder zum anschließenden Berufseinstieg nicht nur den ländlichen Raum, sondern auch die Mittel- und Oberzentren Richtung Groß- und Millionenstädte verlassen. Initiativen, um diese Personengruppe zum Verbleib zumindest im Bundesland zu bewegen, waren bisher wenig erfolgreich.

Es zeigt sich bei den Motiven für die Abwanderung von Berufseinsteigenden, dass diese primär von gut erreichbaren, ausbildungsadäquaten Arbeitsmöglichkeiten und einer guten infrastrukturellen Ausstattung mit reichem Angebot an Gütern, Dienstleistungen, Fortbildungs- und Freizeiteinrichtungen - der Kategorie soziökonomischer Motive mit 
Bezug auf sogenannte „harte“ Standortfaktoren - sowie der Möglichkeit für einen urbanen Lebensstil geprägt sind. Diese Motivstruktur hat mit zunehmender Zentralitätsstufe der Städte eine immer größere Bedeutung (vgl. Abb. 4). Im Vordergrund steht die Arbeitsmigration, die teilweise von Elementen einer „Lifestyle Migration“ überlagert wird. Die politischen und planerischen Möglichkeiten, diese Arbeits- bzw. Lifestylemigration aus den ländlichen Räumen und den niedrigerrangigen Zentren in die höherrangigen Zentren abzuschwächen, sind sehr eingeschränkt.

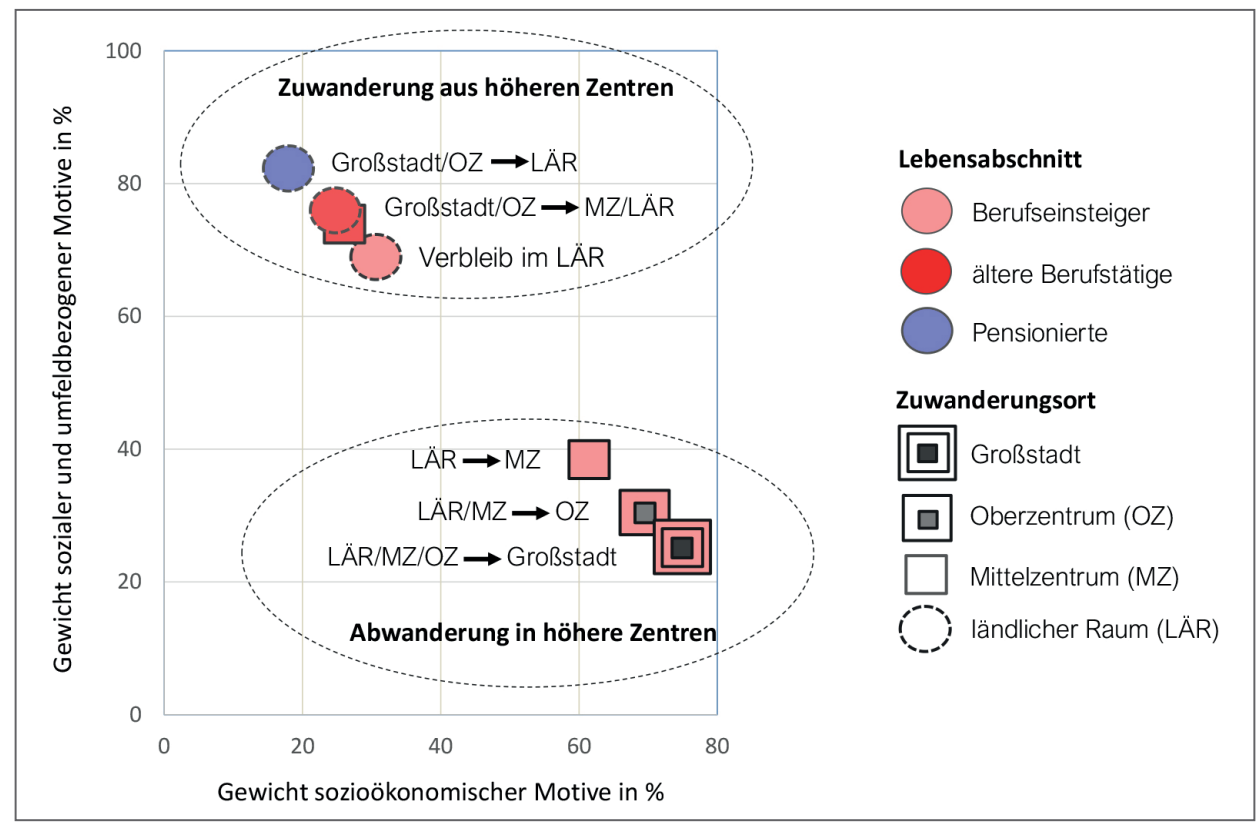

Quelle: Eigene Erhebung und Auswertung (siehe Kapitel 3); eigene Darstellung

Abb. 4: Verhältnis der Gewichtung sozioökonomischer sowie sozialer und umfeldbezogener Motive bei der Migrationsentscheidung in höhere Zentren bzw. aus höheren Zentren

Anders ist die Motivgewichtung bei Zugewanderten aus höheren Zentren in niedrigere Zentren oder den ländlichen Raum sowie Verbliebenen im ländlichen Raum. Sie ist von der Kategorie sozialer und umfeldbezogener Motive mit Bezug auf „weiche“ Standortoder Wohlfühlfaktoren, die subjektiv eine bessere Lebensqualität ermöglichen, wie hohe Umweltqualität, ruhigeres Leben, familiäre Beziehungen und emotionale Ortsbezogenheit, geprägt. Diesen Zusammenhang könnte man auch bis zu einem gewissen Grad für Initiativen zur Verbesserung der Wanderungsbilanz heranziehen.

Eine von vielen derartigen Maßnahmen könnte die Unterstützung oder Neugründung von „Slow Innovations“ sein, wie sie von Heike MAYER (2020) u. a. für Osttirol untersucht wurden. Diese werden vorwiegend von ortsansässigen Unternehmerinnen und Unterneh- 
mern getragen und können sich auch in peripheren Regionen gut behaupten und so einen kleinen, aber stabilen Wirtschaftsfaktor darstellen. Langsame Innovatoren sind oft kleine oder mittelständische Betriebe, deren Innovationsprozesse anders funktionieren als in urbanen Zentren. Sie gehen verstärkt auf kundenspezifische Produkte und Dienstleistungen ein und entwickeln ihre Innovationen eher langfristig, strategisch, oft unter Nutzung einzigartigen lokalen Wissens und im eigenen Bereich ohne häufige und rasche Interaktionen mit anderen.

Die regionalen Zentren (Mittelzentren) im ländlichen Raum bieten offensichtlich erste Ankerpunkte mit einer guten Ausstattung an Arbeits- und Infrastrukturmöglichkeiten, vor allem auch um die strukturellen Defizite im ländlichen Raum auszugleichen. Die Erhaltung und Verbesserung der Ausstattung dieser Zentren kann wahrscheinlich einen Ansatz zum Halten junger Berufseinsteigender aus dem ländlichen Raum, aber auch für ehemals abgewanderte Rückkehrende sein. Den Oberzentren Klagenfurt am Wörthersee und Villach käme in diesem Zusammenhang eine besondere Bedeutung aus der Perspektive des gesamten Bundeslandes zu.

Da die berufs- und arbeitsplatzbezogene Abwanderung kaum zu reduzieren ist, wird der Fokus möglicher Maßnahmen zunehmend auf Möglichkeiten der Zuwanderung in den ländlichen Raum und in die kleineren Zentren zu legen sein. Eine anzusprechende Gruppe könnten potenzielle Rückwanderer sein, weil bei ihnen neben der Umfeldqualität oft auch noch familiäre Beziehungen und eine emotionale Ortsbezogenheit als Wanderungsmotive dazu kommen.

Es erscheint daher sinnvoll, den Kontakt mit den abgewanderten jungen Erwachsenen nicht aufzugeben und für eine allfällige Rückkehr speziell für junge Familien gute Angebote zu bieten. Soziale Kontakte, aber auch die Aufrechterhaltung von Erinnerungen spielen eine wesentliche Rolle für die Rückkehr - ebenso wie Unterstützungsangebote bei der Wohnraum- und Jobsuche, eventuell auch für Partnerinnen und Partner (vgl. JAIN 2009, S. 26ff). Auch gute Bildungs- und Freizeitangebote für Kinder und Jugendliche spielen eine wichtige Rolle und wurden bei der Befragung explizit angesprochen (vgl. auch JAIN 2009, S. 16).

Der große Stellenwert umfeldbezogener Motive kann auch als Hinweis gesehen werden, verstärkt auf „Lifestyle Migration“ zu setzen, wo vielfach eine höhere Lebensqualität in ländlicher Umgebung mit angemessener Infrastrukturausstattung zu günstigeren Kosten als in städtischen Gebieten angestrebt wird. Potenzielle Zuwanderer sind u. a. Personen im Ruhestand, Rückwanderer, Berufstätige als Pendler (eventuell in den letzten Berufsjahren) oder mit zumindest teilweiser Telearbeitsmöglichkeit bzw. Homeoffice, oder auch jüngere Familien in der Phase der Familiengründung, die ihre Kinder in einem ländlichen Umfeld mit hoher Umweltqualität aufwachsen lassen wollen (vgl. GRUBER et al. 2017). Sie können möglicherweise mit einem entsprechenden Angebot zur Zuwanderung motiviert werden.

\section{Literaturverzeichnis}

Aigner-Walder B., Klinglmair R., Leitner S., Sting S. (2015): Ländliche Regionen unter Druck. Zentrale Ergebnisse empirischer Untersuchungen zu Wanderungs-, Bleibe- und 
Rückkehrmotiven bei Wanderungen in und aus Kärnten. Klagenfurt am Wörthersee: Universität Klagenfurt (= IfS Discussion Papers, 04/2015). - https://netlibrary.aau.at/download/ $p d f / 2471615$ (Zugriff: 14.09.2019).

Amt der Kärntner Landesregierung (2013): Sachgebietsprogramm Zentrale Orte. Erläuterungsbericht. Verordnungsentwurf. Klagenfurt am Wörthersee: Land Kärnten.

Amt der Kärntner Landesregierung (2017): Richtlinie zur Abgrenzung des strukturschwachen ländlichen Raumes gemäß Kärntner Wohnbauförderungsgesetz K-WBFG 2017, LGB1. Nr. 68/2017. Klagenfurt am Wörthersee: Land Kärnten.

Barcus H., Halfacree K. (2018): An Introduction to Population Geographies. Lives Across Space. Milton Park / New York: Routledge.

FISCHER T. (2015): Weggehen. Zurückkommen. Verbunden bleiben. Bestimmungsfaktoren des Wanderungsverhaltens junger Menschen im Alter von 20 bis 29 Jahren in der LEADER Region Nationalpark Kalkalpen und Interventionsmöglichkeiten aus raum- und planungswissenschaftlicher Perspektive. Wien: Bundesministerium für Nachhaltigkeit und Tourismus. - https://www.bmnt.gv.at/dam/jcr:28dcd3a0-6892-4986-812a-acb3a66dddf0/04...\% 20\%C2\%B7 (Zugriff: 10.09.2019).

Geist C., McManus P. (2008): Geographical Mobility Over the Life Course: Motivations and Implications. In: Population Space and Place 14 (4), S. 283-303. - https://doi.org/10.1002/psp. 508 (Zugriff: 30.10 .2020 ).

Gruber E., Kobras M., Kordel S. (2017): Lifestyle Migration als Potenzial für ländlich-periphere Gebiete Europas? Die Bewertung wirtschaftlicher und sozialer Auswirkungen aus der Perspektive von Zuwanderern und lokalen Akteuren in Frankreich, Österreich und Spanien. In: Europa Regional, 24 (3-4), S. 16-28. - https://nbn-resolving.org/urn:nbn:de:0168-ssoar57377-7 (Zugriff: 06.11.2020).

JAIN A. (2009): Zu- und Rückwanderung als dynamische Faktoren für ostdeutsche Städte: Ergebnisse der quantitativen Umfragen. - https://www.researchgate.net/publication/282292243_Zuund_Ruckwanderung_als_dynamische_Faktoren_fur_ostdeutsche_Stadte_Ergebnisse_ einer_quantitativen_Studie (Zugriff: 14.11.2020)

Klagenfurt am Wörthersee (2014): Stadtentwicklungskonzept 2020+. Klagenfurt: Magistrat der Stadt Klagenfurt am Wörthersee. - https://www.Klagenfurt am Wörthersee.at/rathausdirekt/aktuelle-projekte/stadtentwicklung-2020.html (Zugriff: 06.01.2020).

Kordel S. (2017): Zuwanderung in ländliche Räume Europas: zur Diversität von Rural Mobilities. In: Europa Regional, 24 (3-4), S. 3-15. - https://nbn-resolving.org/urn:nbn:de:0168-ssoar57309-9 (Zugriff: 02.09.2019).

MAYer H. (2020): Slow Innovation in Europe`s Peripheral Regions: Innovation beyond Acceleration. In: DöRINGER S., EDER J. (eds.): Schlüsselakteure der Regionalentwicklung. Welche Perspektiven bietet Entrepreneurship für ländliche Räume? Wien: Institut für Stadt- und Regionalforschung der ÖAW, S. 9-22 (= ISR-Forschungsberichte, 51). - https://epub.oeaw. ac.at/Oxc1aa5576\%200x003b582d.pdf(Zugriff: 15.11.2020).

ÖAMTC - Österreichischer Automobil-, Motorrad- und Touring-Club: Routenplaner. Wien: ÖAMTC - https://www.oeamtc.at/routenplaner (Zugriff: 22.03.2019).

Österreichische Raumordnungskonferenz (ÖROK) (2018a): Kleinräumige Bevölkerungsprognose für Österreich 2018-2040 mit einer Projektion bis 2060 und Modellfortschreibung bis 2075 (ÖROK-Prognose). Wien: ÖROK. - https://www.oerok.gv.at/fileadmin/user_upload/ Bilder/2.Reiter-Raum_u._Region/2.Daten_und_Grundlagen/Bevoelkerungsprognosen/ Prognose_2018/Bericht_BevPrognose_2018.pdf(Zugriff: 22.09.2019).

Österreichische Raumordnungskonferenz (ÖROK) (2018b): Ergebnisse der ÖREK-Partnerschaft „Strategien für Regionen mit Bevölkerungsrückgang“. Wien: ÖROK (= ÖROK Materialien, 
6). - https://www.oerok.gv.at/fileadmin/user_upload/Bilder/2.Reiter-Raum_u._Region/1. OEREK/OEREK_2011/PS_Bevoelkerung/2018-11-29_Internetversion_BROSCHUERE_ Bevooelkerungsrueckgang.pdf (Zugriff: 17.09.2019)

Österreichische Raumordnungskonferenz (ÖROK) (2019): Anzahl der Arbeitsplätze (Erwerbstätige am Arbeitsort) / 100 EW 2017. In: ÖROK-Atlas Raumbeobachtung. Wien: ÖROK. - https:// www.oerok-atlas.at/\#indicator/23 (Zugriff: 22.09.2019).

Österreichische Raumordnungskonferenz (ÖROK) (2020a): Bruttoregionalprodukt je Einwohner*in: 3-Jahres-Mittel 2014-2016. In: ÖROK-Atlas Raumbeobachtung. Wien: ÖROK. https://www. oerok-atlas.at/\#indicator/29 (Zugriff: 04.10.2020).

Österreichische Raumordnungskonferenz (ÖROK) (2020b): Veränderung der Bevölkerungszahl zu Jahresbeginn 2006-2016 in Prozent. In: ÖROK-Atlas Raumbeobachtung. Wien: ÖROK. https://www.oerok-atlas.at/\#indicator/12 (Zugriff: 04.01.2020).

O`Reilly K. (2014): Lifestyle Migration. Bonn: Bundeszentrale für Politische Bildung (bpb). https://www.bpb.de/gesellschaft/migration/kurzdossiers/198234/lifestyle-migration (Zugriff: 15.11 .2020$)$.

SEGER M. (2019): Österreich - Raum und Gesellschaft. Vermessung der Landschaft, Portraits der Bundesländer. Klagenfurt am Wörthersee: Verlag des Naturwissenschaftlichen Vereins für Kärnten / Österreichische Geographische Gesellschaft.

Statistik Austria (2018): Ein Blick auf die Gemeinde / Gemeindegebarung / Abgaben, Ertragsanteile der Gemeinden. Wien: Bundesanstalt Statistik Austria. - https://www.statistik.at/blickgem/ G0903/g20501.pdf (Zugriff: 22.09.2019).

Statistik Austria (2019a): Bevölkerungsveränderung nach Komponenten 2018 und 2017. Wien: Bundesanstalt Statistik Austria. - https://www.statistik.at/blickgem/G0204/g20901.pdf (Zugriff: 22.09.2019).

Statistik Austria (2019b): Übernachtungen pro Einwohner. Wien: Bundesanstalt Statistik Austria. https://www.statistik.at/atlas/ (Zugriff: 22.09.2019)

Stockdale A. (2016): Contemporary and 'Messy' Rural In-migration Processes: Comparing Counterurban and Lateral Rural Migration. In: Population Space and Place, 22 (6), S. 599-616. - https://doi.org/10.1002/psp.1947 (Zugriff: 30.10.2020).

Thомаs M. (2019): Employment, Education, and Family: Revealing the Motives Behind Internal Migration in Great Britain. In: Population Space and Place 25 (4), article e2233. - https:// doi.org/10.1002/psp.2233 (Zugriff: 30.10.2020).

Wingens M. (2020): Soziologische Lebenslaufforschung. Wiesbaden: Springer. 